\title{
Investigating the Mucoid Switch of Pseudomonas aeruginosa at Oil-Water Interfaces
}

Stephen D. House ${ }^{1,2 *}$, Sricharani Rao Balmuri ${ }^{1}$, Judith C. Yang ${ }^{1,2,3}$ and Tagbo H.R. Niepa $a^{1,4,5,6 *}$

${ }^{1 .}$ Department of Chemical and Petroleum Engineering, University of Pittsburgh, Pittsburgh, PA, USA.

2. Environmental TEM Catalysis Consortium (ECC), University of Pittsburgh, Pittsburgh, PA, USA.

3. Department of Physics and Astronomy, University of Pittsburgh, Pittsburgh, PA, USA.

4. Department of Bioengineering, University of Pittsburgh, Pittsburgh, PA, USA.

5. Department of Civil and Environmental Engineering, University of Pittsburgh, Pittsburgh, PA, USA.

6. Center for Medicine and the Microbiome, University of Pittsburgh, Pittsburgh, PA, USA.

*Corresponding authors: sdh46@pitt.edu and tniepa@pitt.edu

Cystic fibrosis is a genetic disorder caused by a mutation in the CFTR transporter protein that induces a build-up of mucus along the linings of various internal organs. Homeostasis of electrolytes through the epithelial cells is lost leading to the thick mucus in the lining of lungs, which in chronic cases often leads to death from obstructed breathing. This environment also serves as a niche for bacterial cells to colonize and form biofilms and lead to recurring infection. Pseudomonas aeruginosa is one such persistent bacteria found in most of the cystic fibrosis infections. Two phenotypes of $P$. aeruginosa have been isolated from cystic fibrosis patients - mucoid (PASL) and non-mucoid (PANT) strain [1] - which are distinguished by their ability to produce different compositions of exopolysaccharide (EPS) [2]. PASL strains produce a thick and slimy EPS which is high in alginate content due to over expression of mucA gene, compared to that of PANT. In cystic fibrosis, non-mucoid strains of $P$. aeruginosa colonize the lungs whereupon a prevalent mutation in anti-sigma factor mucA has been observed to lead to a switch into the mucoid strain [3]. Mucoid switch is thought to enable $P$. aeruginosa survive through the hypoxic environments by secreting high amounts of EPS. It is also proposed that transition to mucoid strain protects the bacteria from phagocytosis by forming microcolonies enclosed in a highly thick matrix of EPS. We hypothesize that the microbes undergo this mucoid switch, facilitating increased EPS production, in order to cope with the interfacial stresses present at the fluid interface, increasing their chances of survival. Designing effective treatments for infections associated with these persistent bacteria requires a better understanding of the role played by their interfacial films, including how their structure, composition, and evolution impacts the survivability of the bacteria.

In this work, we present our study of the biofilms produced by these two strains and the impact on their survival at the fluid interface. We selected the hexadecane-water system to study the interfacial films in order to compensate for the evaporation of oil and because its transparency allows for better observation of the structural deformations of the films. Pendant drop tensiometry was employed to measure the interfacial stresses present [4]. Bacterial isolates were exposed to the hexadecane-water interface of 20 $\mu \mathrm{L}$ droplets, for a period of 24 hours, allowing them to form interfacial films. As the cells adsorb to the surface, the interfacial tension decreases over time until a constant value is reached (Fig. 1a). Due to the presence of the slimy layer around the cells and the composition of their EPS, a steep drop in the interfacial tension was observed for the mucoid strain (PASL) when compared to that of the non-mucoid (PANT).

These interfacial films were then collected on titanium foils. Water from the droplet was carefully removed and the foil with the film was fixed in gluteraldehyde (overnight). Samples for SEM investigation - using a Zeiss Sigma 500 VP FE-SEM operated at $5 \mathrm{keV}$ - were prepared by treating the 
samples with HEPES buffer and a series of alcohol washes before being drop-cast and coated with $3 \mathrm{~nm}$ of Au-Pd. The SEM images (Fig. 1b-e) reveal significant differences in the morphological changes in the interfacial microbial films. The PASL bacteria (Fig. 1b) exposed to the interfaces retained their intact cellular morphology, whereas PANT bacteria (Fig. 1d) exhibited distorted morphologies, including large numbers of lysed cells. This indicates that the mucoid coating of the PASL plays an important role in protecting the bacteria from the interfacial stresses. Related, the structure of the film itself was also found to vary. The microbes in the PASL films were densely packed and highly connected (Fig. 1c), unlike the PANT films which exhibited a stringy, sparser arrangement (Fig. 1e). Interestingly, it appeared that the lysed PANT cells themselves were incorporated into the film and may form a sacrificial buffer layer to protect PANT cells deeper in the colony. Transmission electron microscopy (TEM) experiments are underway to probe the morphological differences between strains at the interface with higher resolution and without the need for metalized coatings. Scanning TEM spectroscopy is being used to determine the composition of the biofilms and confirm the presence and destination of the cellular material from the lysed PANT cells. We are also exploring alternative fixing methods, to decouple any effects of specimen preparation and amplify the structural impact of the interface. Understanding the effect of interfaces on the phenotypical changes of cells provides information on disease prognosis thereby allowing us to take preventive measures to avoid chronic infection, which is otherwise difficult to treat [5].

\section{References:}

[1] AY Bhagirath et al., BMC Pulmonary Medicine 16 (2016), p. 174.

[2] B Pritt, L O'Brien and W Winn, American Journal of Clinical Pathology 128 (2007), p. 32.

[3] RL Henry, CM Mellis and L Petrovic, 12 (1992), p. 158.

[4] THR Niepa et al., Scientific Reports 7 (2017), p. 17864.

[5] This work is supported by NSF-DMREF through contract No. CHE-1534630 (SDH, JCY) and Niepa Laboratory start-up funds (SRB, THRN) provided by the U. Pitt Swanson School of Engineering. The microscopy was performed at the Nanoscale Fabrication and Characterization Facility (NFCF) at U. Pitt. The ECC is funded through U. Pitt and Hitachi High Technologies. The authors would like to thank Dr. Kevin Alby (U.Penn) for providing the bacterial isolates. Stephen D. House and Sricharani Rao Balmuri contributed equally to this work
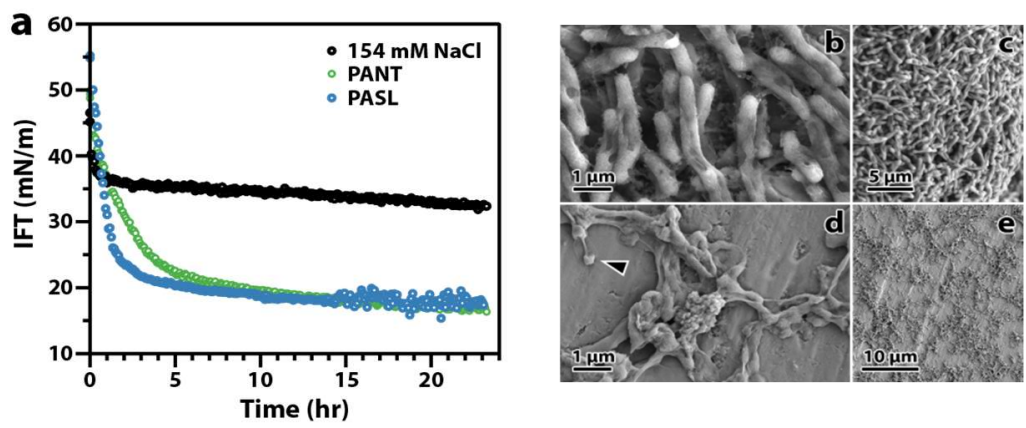

Figure 1. (a) Changes in dynamic interfacial tension as a function of time, measured by pendant drop tensiometry, of mucoid and non-mucoid strains at the hexadecane-water interface. (b-e) SEM images of $(b, c)$ mucoid/PASL and $(\mathrm{d}, \mathrm{e})$ non-mucoid/PANT films formed at the hexadecane-water interface. The arrowhead indicates an example lysed PANT cell. 